\title{
Research Paper \\ Locomotive Syndrome in the Elderly and Its Risk Factors
}

\author{
Nasim Sadeghimahalli ${ }^{1}$, Mohammad Ali Hosseini ${ }^{2} \odot$, Mehdi Rahgozar ${ }^{3} \odot,{ }^{*}$ Kian Norouzi Tabrizi $^{1} \odot$
}

1. Iranian Research Center on Aging, University of Social Welfare and Rehabilitation Sciences, Tehran, Iran.

2. Department of Rehabilitation Management, University of Social Welfare and Rehabilitation Sciences, Tehran, Iran.

3. Department of Biostatistics, University of Social Welfare and Rehabilitation Sciences, Tehran, Iran.

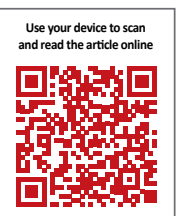

Ceftration Sadeghimahalli N, Hosseini MA, Rahgozar M, Norouzi Tabrizi K. [Locomotive Syndrome in the Elderly and Its Risk Factors (Persian)]. Salmand: Iranian Journal of Ageing. 2019; 13(Special Issue):604-613. https://doi.org/10.32598/SIJA.13.Special-Issue.604

https://doi.org/10.32598/SIJA.13.Special-Issue.604

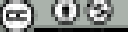

Received: 12 May 2018

Accepted: 17 Oct 2018

Available Online: 10 Mar 2019

Key words:

Locomotive Syn-

drome, Logistic re-

gression, Risk factors,

Elderly

\section{A B S T R A C T}

Objectives Locomotive Syndrome (LS) refers to reduced mobility due to the impairment of locomotive organs. LS results in movement limitation, loss of balance, pain sensation, muscle weakness, and ultimately increased demand for long-term nursing care. This study aimed to investigate LS in Iranian elderly and its risk factors.

Methods \& Materials This descriptive, cross-sectional study was conducted on randomly selected 320 community-dwelling elderly people in Qaem Shahr City, Iran, in 2018. The study was approved by the Ethics Committee of the University of Social Welfare. The study instruments were demographic form and geriatric locomotive function scale-25 Persian questionnaire. The obtained data were presented by descriptive statistics and logistic regression was used to investigate the correlation between the criterion and predictor variables. All analyses were done in SPSS-V23.

Results The Mean \pm SD age of the participants was $69.85 \pm 7.7$ years. About $58 \%$ of the participants were in the LS group. Variables of age ( $O R=1.05$, increase per 1 year), female gender $(O R=2.7)$, positive history of chronic disease $(O R=3.4)$, and positive history of chronic pain $(O R=4.04)$ were the predictors of $L S$ among elders.

Conclusion Regarding the high prevalence of LS and its relationship with demographic factors (age and sex) as well as chronic disease and, chronic pain, screening methods to identify those at risk for LS and taking preventive measures are highly recommended.

\section{Extended Abstract}

\section{Objectives}

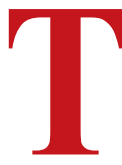

he increase in life expectancy is the inevitable result of development in the health sector [1]. By 2050, the number of Iranian elders will be quintupled [2, 3]. Aging affects different aspects of life (Activities of daily living, mobil- ber of people who need nursing care will increase, which results in financial, social, and health burden on society $[5$, 6]. Orthopedic disorders are the main reasons for calling nursing services [7, 8]. In 2007, the Japanese Orthopedic Association introduced the term Locomotive Syndrome (LS) [9]. LS is the condition of reduced mobility due to impairment of locomotive organs $[10,11]$. LS results in a limitation in movement, imbalance, pain sensation, muscle weakness, and high demand for long-term nursing care [12, 13]. Early detection of the risk factors can help prevent this ity, health status) [4]. Also, with population aging, the num-

\section{* Corresponding Author:}

Kian Norouzi Tabrizi, PhD.

Address: Iranian Research Center on Aging, University of Social Welfare and Rehabilitation Sciences, Tehran, Iran.

Tel: +98 (21) 22180154

E-mail: ircauswr@gmail.com 
condition. This study aimed to investigate LS in Iranian elderly and its risk factors.

\section{Methods and Materials}

This descriptive, cross-sectional study was conducted on randomly selected 320 community-dwelling elderly in Qaem Shahr City, Iran, in 2018. The study was approved by the Ethics Committee of the University of Social Welfare.

The study inclusion criteria were individuals $\geq 60$ years old of either sex, ability to check and answer questionnaires (checked by AMT), and willingness to participate in the research. The exclusion criteria were an inability to walk without assistance, having severe neurologic, cardiovascular, pulmonary or renal disease, suffering from mental illness, history of fractures of the lower extremities and or spine within the last 6 months, and receiving treatments for acute trauma.

The study tools were the demographic questionnaire and the Persian version of geriatric locomotive function scale-25 (Persian-GLFS25). The demographic questionnaire required information about study participants' age, gender, marital status, education, job, falling, and positive history for chronic pain/diseases. The Persian-GLFS25 contains 25 questions; 4 questions for pain assessment, 17 questions related to daily activities and the quality of life of the elderly, and 4 questions related to social and psychological functioning. Each item is rated from 0 to 4 based on a Likert-type scale. The psychometric properties of the tool in Iranian elderly have been assessed and confirmed. The reliability of the scale was assessed by test-retest and internal consistency (The Cronbach alpha) and calculated results were 0.84 and 0.93 , respectively $(\mathrm{P}=0.01)$. The con- current criterion validity was conducted between GLFS25 and EQ-5D (European Quality of Life-5 dimensions) and its Pearson correlation coefficient was found as 0.86 . The cut-off score for diagnosis of LS was set at 16 (sensitivity $=0.88$, specificity $=0.84$ ) in Iranian elders [14]. The obtained data were presented with descriptive statistics. The correlation between the LS and predictor variables were investigated by logistic regression. All statistical analyses were done in SPSS.V 23.

\section{Results}

The Mean \pm SD age of the samples was $69.85 \pm 7.7$ years. About 55\% were in the age category of 60-69 years. Also, $66 \%$ of the samples were male, $75 \%$ were married, and $86 \%$ had a diploma or under diploma literacy level. More than $51.5 \%$ of the participants were retired. About $78 \%$ of the samples have at least 1 chronic disease. Cardiovascular diseases were the most common illness $(77.8 \%)$. About $49 \%$ percent had a positive history of chronic pain, and $57.5 \%$ had a positive history of falling, at least once, in the past year.

Of the study samples, $42 \%$ were in the non-LS group and $58 \%$ in the LS group. The Mean \pm SD scores of GLFS- 25 questionnaire in the LS group and the non-LS group were $30.5 \pm 13.25$ and $8.52 \pm 4.4$, respectively and this difference was statistically significant $(\mathrm{P}<0.001)$.

To predict the outcomes of LS among 320 elders, the logistic regression analysis was used. The final model explained $28.9 \%$ to $38.9 \%$ of the total variance. The model was fitted with data (Hosmer-Lemeshow test, $\chi^{2}=5.16$, $\mathrm{P}=0.74$ ) and was able to predict the status of the locomotive syndrome (Omnibus tests, $\chi^{2}=109.29, \mathrm{P}<0.001$ ). Finally, the model properly predicted $75 \%$ of the cases. Seven

Table 1. Estimation of parameters of the logistic regression model

\begin{tabular}{|c|c|c|c|c|c|}
\hline Predictive Variables & Regression Coefficient & Wald 2 & OR & Cl for $\operatorname{Exp}(B)$ & $\mathbf{P}$ \\
\hline Age & 0.05 & 6.64 & 1.056 & 1.013-1.101 & $0.010^{*}$ \\
\hline Gender (Female) & 0.98 & 8.50 & 2.66 & $1.379-5.153$ & $0.004^{* *}$ \\
\hline Positive history of chronic disease & 1.23 & 12.15 & 3.425 & $1.714-6.842$ & $<0.001^{* *}$ \\
\hline Positive history of chronic pain & 1.39 & 21.35 & 4.046 & $2.237-7.320$ & $<0.001^{* *}$ \\
\hline Marital status (divorced-widow) & 0.77 & 3.20 & 2.155 & $0.930-4.994$ & 0.073 \\
\hline The level of literacy (diploma and under) & 0.42 & 1.12 & 1.517 & $0.701-3.282$ & 0.290 \\
\hline Positive history of falling in the past year & -0.25 & 0.70 & 0.776 & $0.430-1.401$ & 0.401 \\
\hline
\end{tabular}


predictor variables were entered the model, by ENTER method and 4 variables successfully predicted the status of LS. The results showed that the variables of age, gender, positive history of chronic disease and chronic pain significantly predicted the LS $(\mathrm{P}<0.05)$. Variables of age $(\mathrm{OR}=1.05$, increase per 1 year, $\mathrm{CI}=1.01-1.10)$, female gender $(\mathrm{OR}=2.7, \mathrm{CI}=1.38-5.15)$, positive history of chronic disease $(\mathrm{OR}=3.4, \mathrm{CI}=1.71-6.84)$ and positive history of chronic pain $(\mathrm{OR}=4.04, \mathrm{CI}=2.23-7.32)$ predicted $\mathrm{LS}$ among elders (Table 1). Linear and multi-linear assumptions were also examined.

\section{Conclusion}

The results showed that the diagnosis of locomotive syndrome has a strong association with some variables. For example, our study showed that female gender increases the risk of LS up to 5 times. A positive history for chronic pain and chronic diseases increases the odds for LS, 4 and 3.5 times, respectively. Studies by Kimura et al. (2014), Ebihara et al. (2013), Kawaguchi et al. (2014), Youshimura and Nakamura (2016), Sasaki et al. (2012), Nakamura et al. (2016), Chiba et al. (2016), Muramoto et al. (2016), and lizuka et al. (2015) support our results, too [15-22]. Regarding the high prevalence of LS in the elderly and its known correlation with these factors, screening the older people who are at risk for LS and taking proper precautions are highly recommended. In the future, nurses should play a more proactive role and provide services such as preventive and supportive measures, client teaching, counseling and so on in this regard.

\section{Ethical Considerations}

Compliance with ethical guidelines

The research was performed according to general ethical guidelines. It obtained the necessary permissions from the Ethics Committee of Tehran University of Social Welfare and Rehabilitation Sciences (IR.USWR.REC.2017.46).

Funding

This study was funded by the Iranian Research Center on Aging of University of Social Welfare and Rehabilitation Sciences, Tehran, Iran.

Authors' contributions

All authors contributed in designing, running, and writing all parts of the research.

\section{Conflicts of interest}

The authors declared no conflict of interest.

\section{Acknowledgments}

We thank all professors who assisted us in this research and especially and Mr. Reza Ghanei for his comments that greatly improved the manuscript. 


\title{
سندرم حركتى در سالمندان قائمششهر و عوامل خطرساز آن
}

نسيم صادقى محلى' ه محمدعلى حسينى' •، مهدى رهكذر' ه •كيان نوروزى تبريزى'

1- مركز تحقيقات سالمندى، دائشكاه علوم بهزيستى و توانبخشى، تهران، ايران.

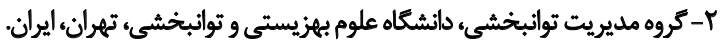

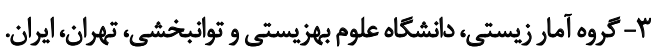

\begin{abstract}
حكين

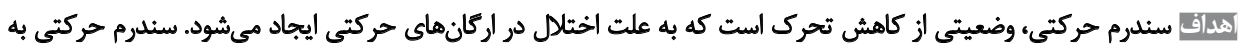

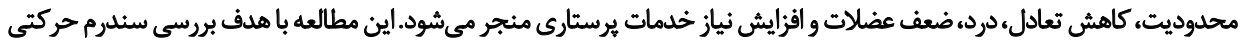

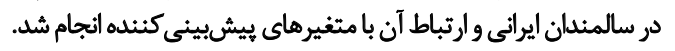

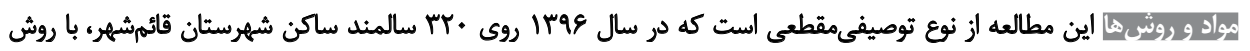

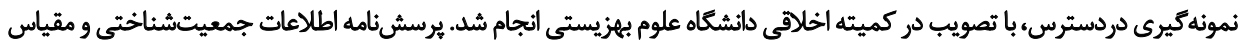

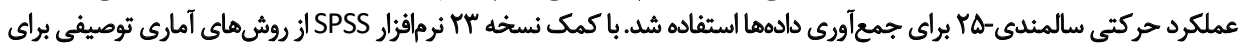

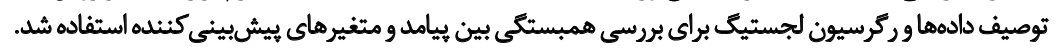
بإقته ها) ميانكين سنى نمونهها

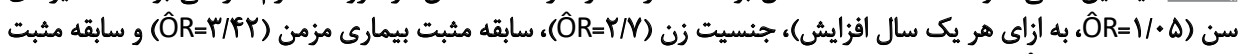

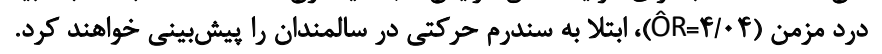

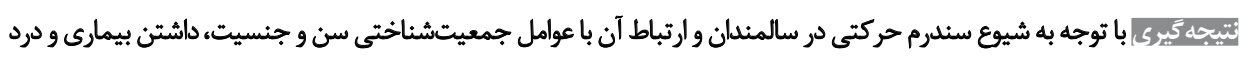

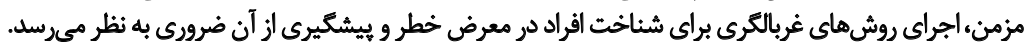

تاريخ دريافت:Trارديبهشت

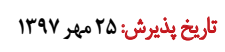
تاريخ انتشار:19 اسفند 19

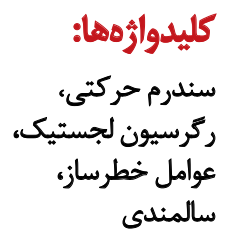

يكى از جالشهاى دوره سالمندى مشكلات حركتى سالمندان

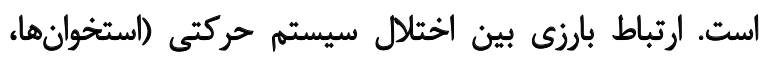

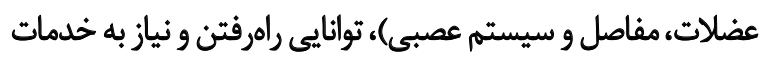

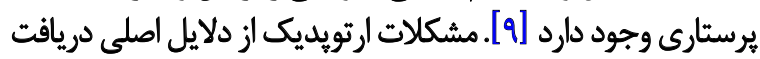

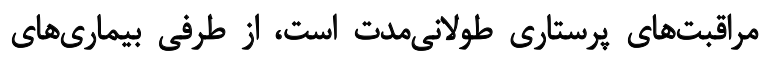

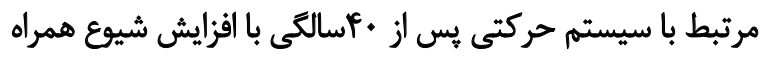

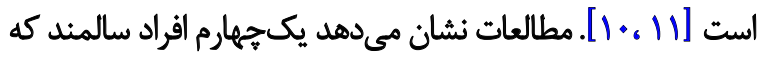

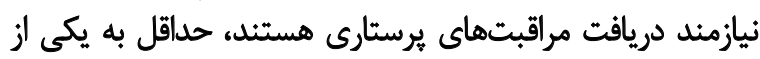

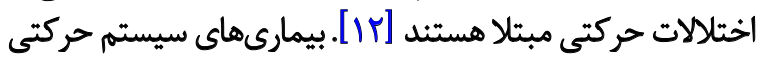

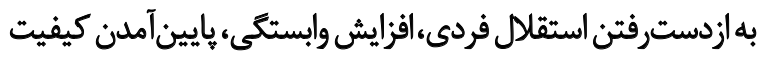

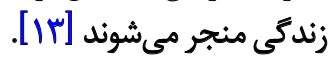

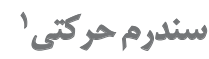

مفهوم سندرم حركتى، در سال V •. ب براى شناسايى سريعتر

1. Locomotive Syndrome

\section{a.}

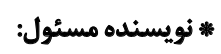

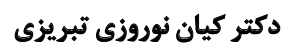

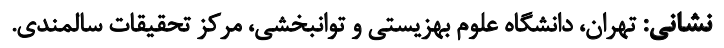

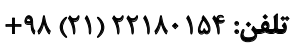
يست الكترونيكي: ircauswr@gmail.com

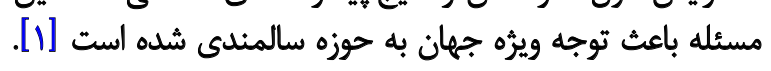

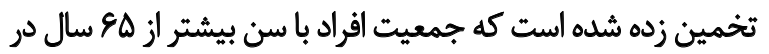

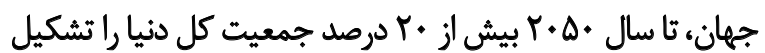

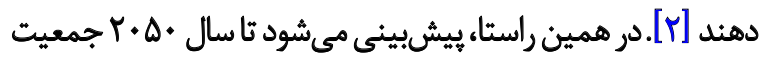

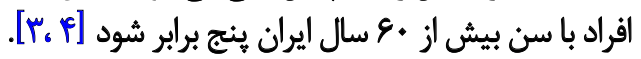
افزايش طول عمر بر جنبههاى مختلف زند زئى سالمند هميجون

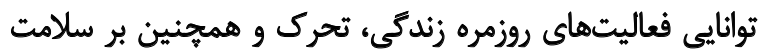

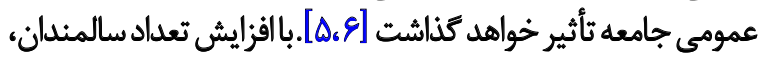

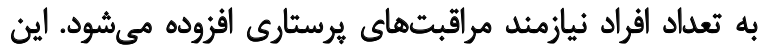

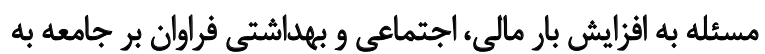

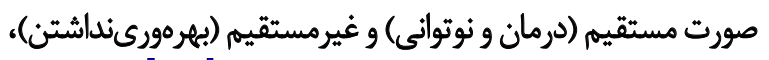

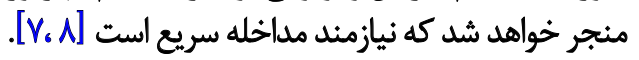




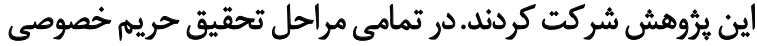

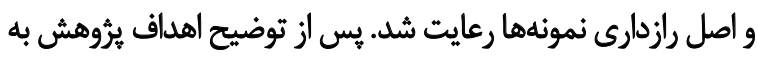

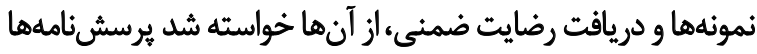

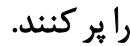

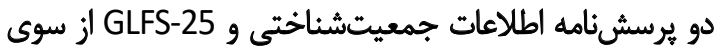

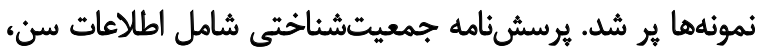

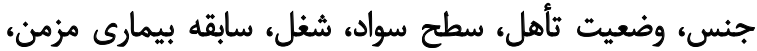

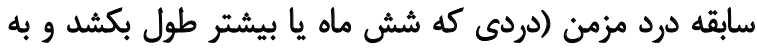

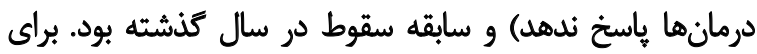

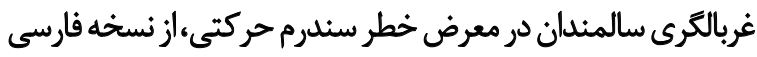

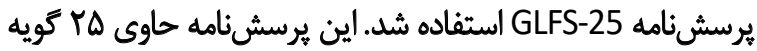

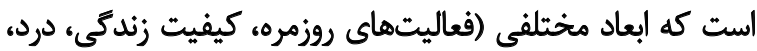

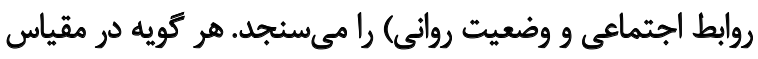
صفر تا F امتياز نمره تخذارى مىشود.

نمره نهايى يرسشنامه بين صفر تا . . أست. نقطه برش ابزار

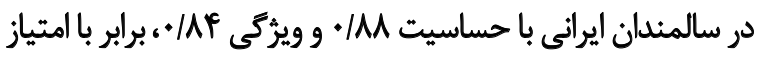

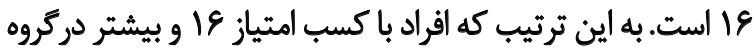

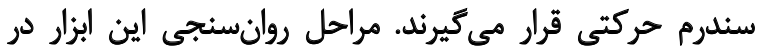

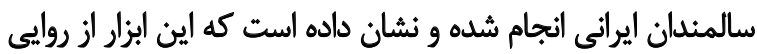

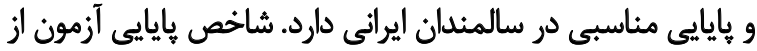

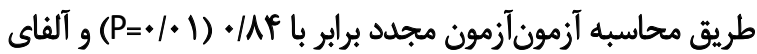

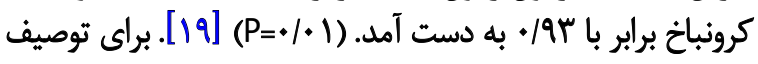

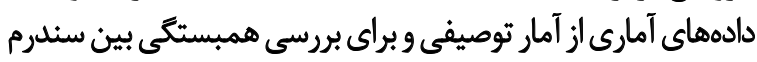

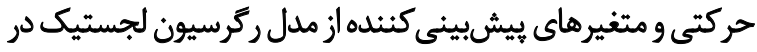
محيط نرم|فزار SPSS نسخه برى استفاده شد

ياقتتهها

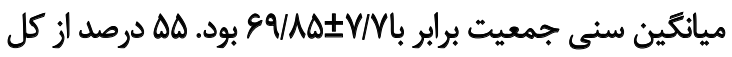

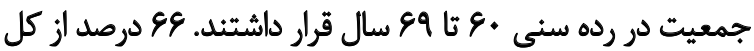

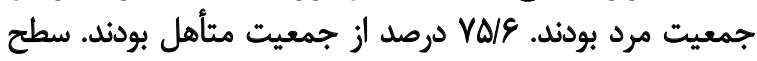

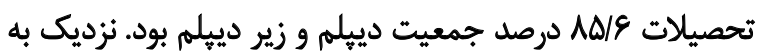

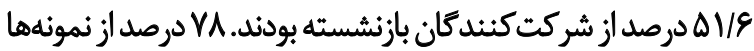

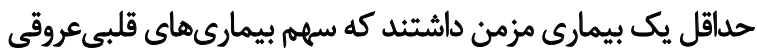

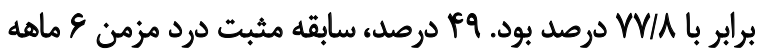

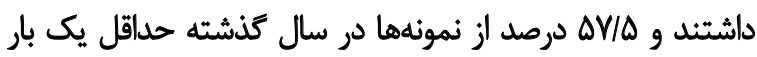

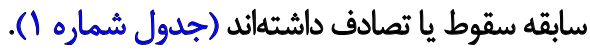

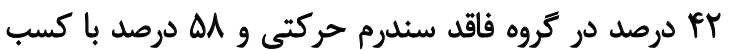

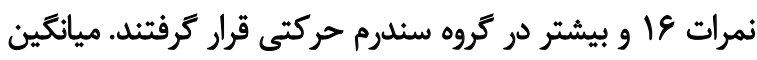

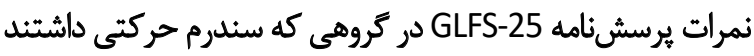
و كروهى سندرم حركتى نداشتند به تروتيب براي

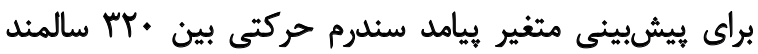

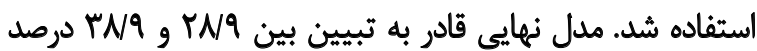

سالمندان در معرض خطر و كاهش تعداد سالمندان نيازمند

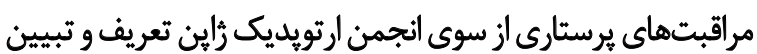

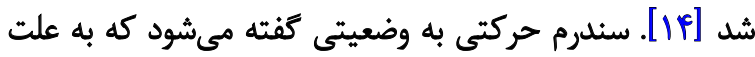

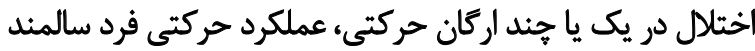

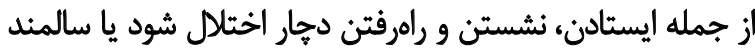

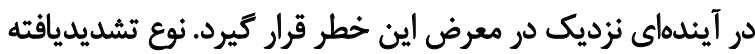

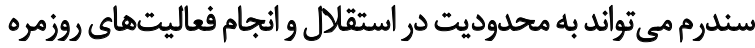

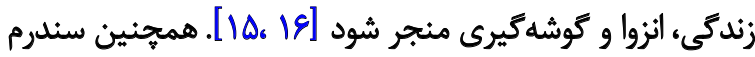

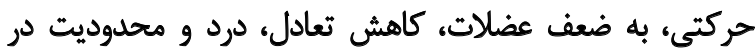

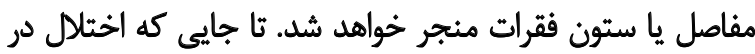

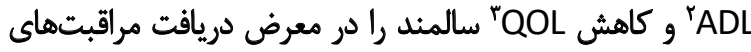

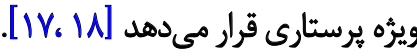

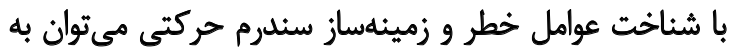

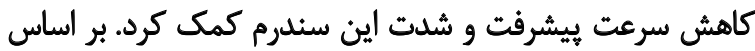

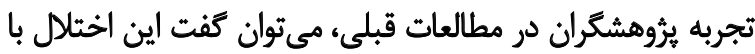

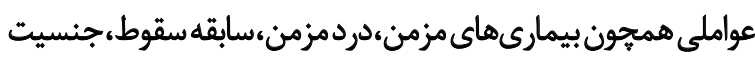

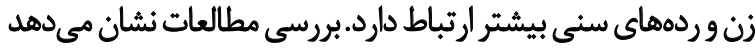

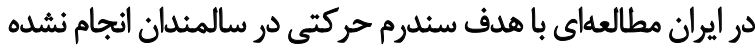

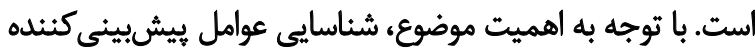

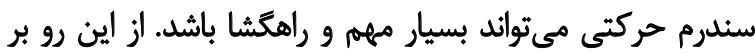

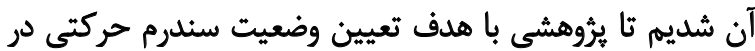

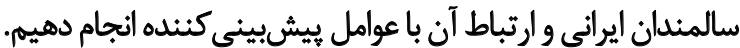

روش مطالعه

اين مطالعه از نوع توصيفىمقطعى است كه به صورت مقطعى

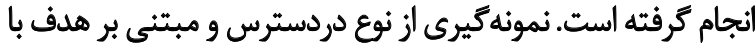

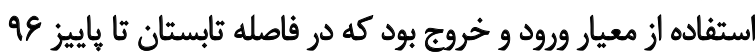

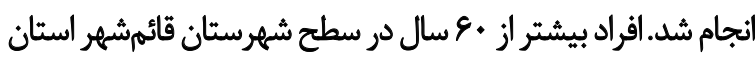
مازندران نمونه يُروهش را تشكيل مي دادند.

معيارهاى ورود عبارتند ازي: زنان و مردان بيشتر از •9 سال، افراد

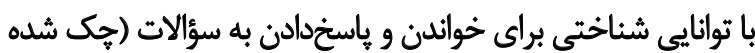

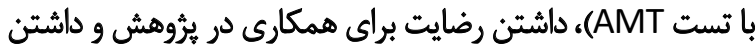

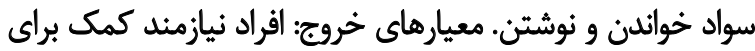

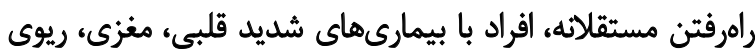

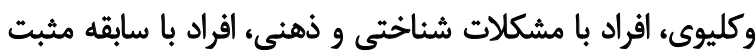

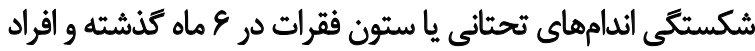

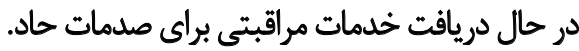

حجم نمونه با توجه به طراحى يُّوهش از نوع همبستئكى تعداد

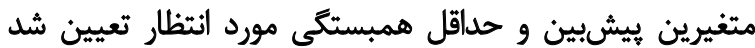

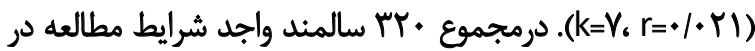

2. Activities Daily of Living

3. Quality of Life 
جدول ا. توزيع فراوانى نمونهها بر حسب متغيرهاي مطالعه

\begin{tabular}{|c|c|c|}
\hline \multirow{2}{*}{ فراوانى (د,صد) } & \multicolumn{2}{|c|}{ متغيرها } \\
\hline & g. - 9 & \\
\hline$N A(Y \& / 8)$ & $g Q-q q$ & \\
\hline$\Delta)(1 \Delta / 9)$ & $V \cdot-V^{\varphi}$ & سن \\
\hline$\Delta \cdot(1 \otimes / 8)$ & $V D-V q$ & \\
\hline$m \mu(1 r / F)$ & ي. & \\
\hline $\operatorname{rir}(\xi g / M)$ & مرد & \\
\hline $1 \cdot A(M / A)$ & is & جنسيت \\
\hline$I r(r / \Lambda)$ & هركَز ازدواج نكرده & \\
\hline $\operatorname{rer}(\mathrm{V} / \mathrm{g} / \mathrm{s})$ & داراي همسر & وضعيت تأهل \\
\hline$g q(Y \cdot / g)$ & مطلقه//ييوه & \\
\hline $\operatorname{Tr}^{2}(\Lambda \Delta / C)$ & دييلم و زير دييلم & \\
\hline$r \cdot(g / \pi)$ & فوق دييلم & سطح سواد \\
\hline$r q(N))$ & ليسانس به بالا & \\
\hline$W(\Delta / 9)$ & كار دولثي & \\
\hline$\Delta P(I V / \Delta)$ & آزاد/كاركر & \\
\hline $18 \Delta(\Delta / / 9)$ & بازنتسته & وضعيت اشتغال \\
\hline$M(\Delta / \&)$ & كشاوزز & \\
\hline $\operatorname{Rr}(19 / V)$ & خائهدار & \\
\hline $\operatorname{req}(W / A)$ & ماشتيند & \\
\hline$n(r / T)$ & نداششتند & سابقه ييمارى مزمن \\
\hline $\operatorname{lon}\left(\operatorname{coq}^{e}\right)$ & ماشتئد & \\
\hline $18 r(\Delta \cdot 19)$ & ندائشتند & سابقه درد مزمن \\
\hline $\operatorname{Irg}(T / T)$ & ن نداشتيند & \\
\hline$q\left(r_{+}\right)$ & البار & سابقه سقوط در يك سال كذشته \\
\hline$\Delta r(1 \& / M)$ & كبار & \\
\hline$m e(11 / \pi)$ & لا لو بيشتر & \\
\hline
\end{tabular}

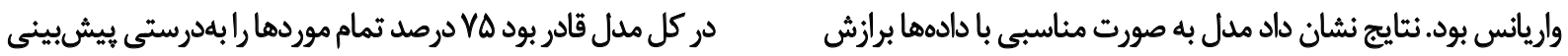

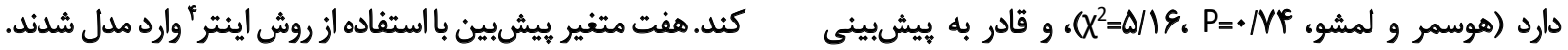
4. Enter

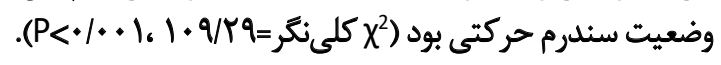


جدول r. ميائكين نمرات يرسشنامه در جمعيت

\begin{tabular}{|c|c|c|c|c|}
\hline انحراف معيار & 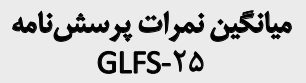 & تعداد (درصد) & & متّغير \\
\hline $10 / 1$. & TIMT & MT. & & كل نمونه \\
\hline Ir/S/A & $\begin{array}{l}\text { TVEF } \\
\text { rV/ar }\end{array}$ & $\begin{array}{l}99(\%)) \\
N \&(r V)\end{array}$ & زرد & كروهي كه سندرم حركتى دارند. \\
\hline $\begin{array}{l}\text { P/PQ } \\
\text { T/R }\end{array}$ & $\begin{array}{l}N \cdot 9 \\
1.19 .\end{array}$ & $\begin{array}{l}\| r(r \Delta) \\
r r(y)\end{array}$ & زمن & كروهي كه سنلرم حركتى ثلارند. \\
\hline
\end{tabular}

2

جدول ب. برآورد بارامترهاي مدل لجستيك ركرسيون

\begin{tabular}{|c|c|c|c|c|c|}
\hline مقدار الحتمال & فاصله اطمينان & نسبت شانس & آماره والد & ضريب ركريسيون & مثغير هاني ييشبين \\
\hline .1 .1 & $1 / \cdot 1 m-1 / 1 \cdot 1$ & $V \cdot \Delta s$ & elef & $.1 \%$ & سن سن \\
\hline $.10+4$ & I/Trq-ه//Ar & r/ge & Na. & ./4 & جنسيت زن \\
\hline$<+1+\infty$ & IMIF-S/AFT & W/FTO & $I r / N \Delta$ & V & سابقه مثبت بيمارى مزمن \\
\hline$<+1+\infty$ & $V / T H \cdot-T / T H V$ & $p /+48$ & $r / / r \Delta$ & $1 / 79$ & سابقه مثبت درد مزمن \\
\hline$\cdot 1 \cdot n^{n}$ & $. / 94 \cdot-7 / 997$ & $r / 1 \Delta \Delta$ & $M / T$. & $\cdot M$ & تأهل (مطلقة/ بيوه) \\
\hline.$/ 79$. & . M.I-r/rar & V/DIV & I/T & . /er & سطح سواد دييلم و زير دييلم \\
\hline$. / 4+1$ & $1 / 4 \cdot 1-\cdot / 4+$ &. We & $\cdot / \mathrm{V}$ &.$- / T \Delta$ & سابقه مثبت سقوط در يك سال كذشته \\
\hline
\end{tabular}

ناتوانى هاى سالمندان در آينده بيشگيرى كرد. به علت نوظهوربودن

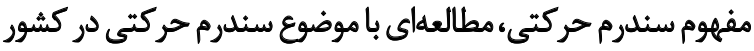

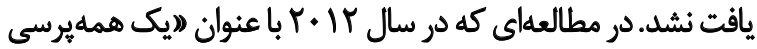

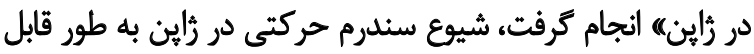

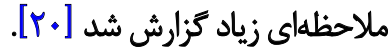

نتايج اين بثروهش نشان مى دهد احتمال تشخيص و بروز سندرم

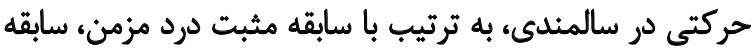

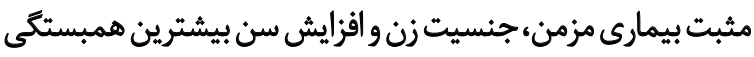

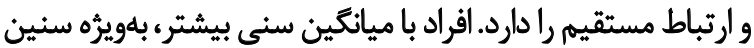

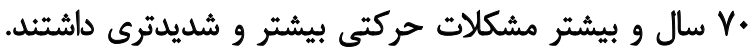

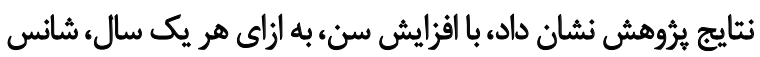

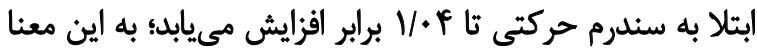

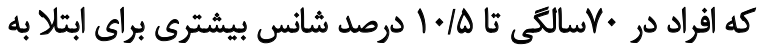

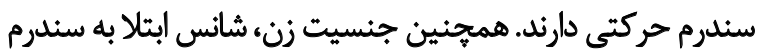

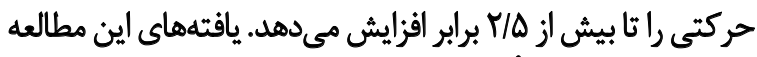

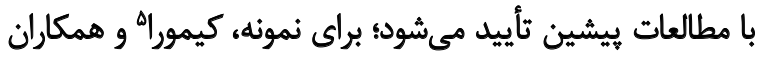

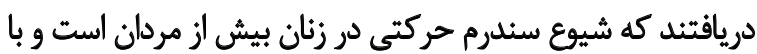

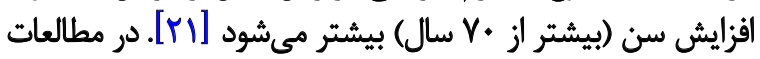

\section{Kimura}

جهار متغير از بين آنها به صورت موفقيتآميزى وضعيت سندرم حركتى را بيشبينى مي أنرد.

نتايج نشان داد متغير هاى سن، جنسيت زن، داشتن سابقه مثبت

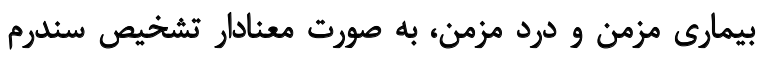

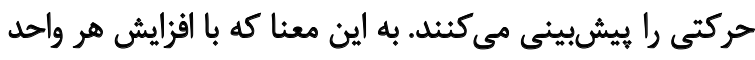

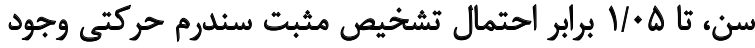

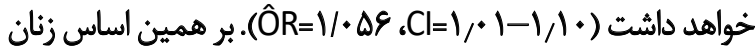

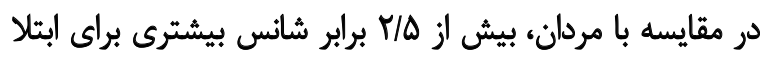

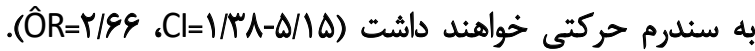

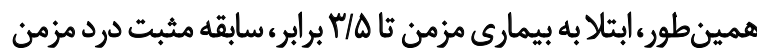

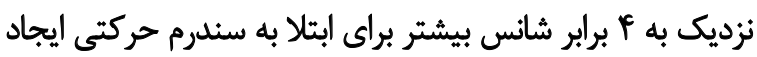

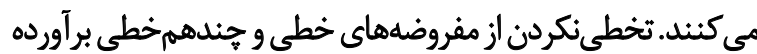
شده بود (جدول شماره س).

ث๐

اين ثئوهش با هدف بررسى وضعيث سندرم حركتى و عوامل

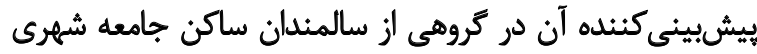

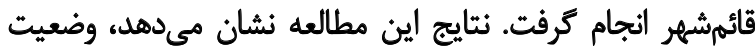

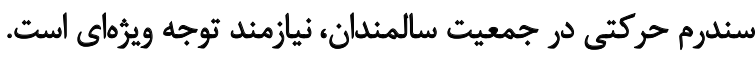

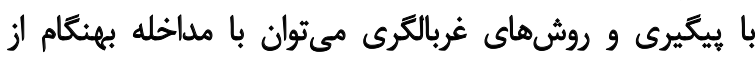


نظر مىرسد فقدان أكاهى أز آن، در جمعيت عمومى سالمند وجود

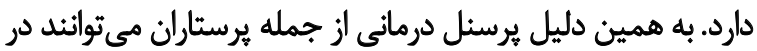

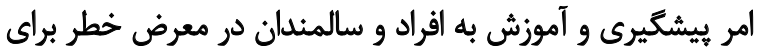
كاهش و درمان عوامل خطرساز نقش فعالى داشته باشند.

از سوى ديكر، شناسايى سالمندان در معرض خطر سندرم

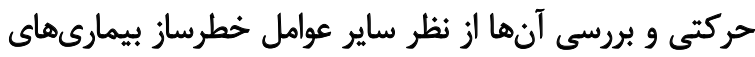

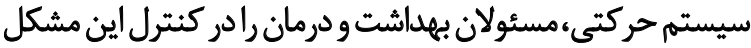

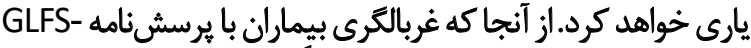

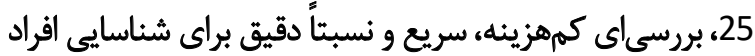

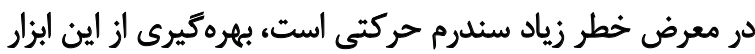

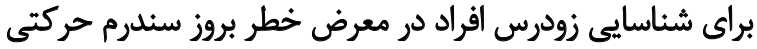

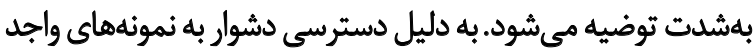

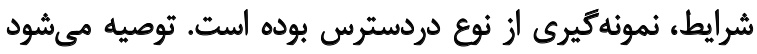

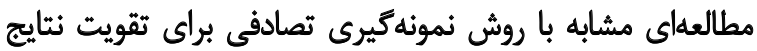

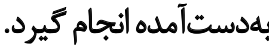

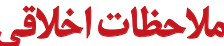

\section{يبيروى از اصول اخلاق يثوهش}

اين مطالعه مجوزهاى لازم را از كميته اخلاق دانشكاه علوم بهزيستى و توانبخشى تهران (IR.USWR.REC.2017.46) كرفته علائ

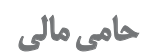

مركز تحقيقات سالمندى ايران در دانشكاه علوم بهزيستى و توانبخشى تهران از اين مطالعه حمايت مالى كرده است.

$$
\text { مشاركت نويسندكان }
$$

تمام نويسندكان در طراحى، اجرا و نتارش همه بخشهاى ئروهش حاضر مشاركت داشتهاند.

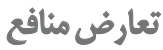

بنا به اظهار نويسندكان، در اين مقاله هيجكونه تعارض منافعى

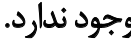

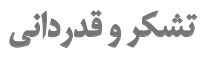

بدين وسيله از همكارى و حمايتهاي بىدريغ آقاى دكتر رضا

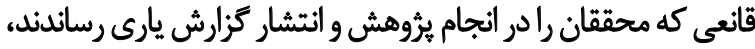

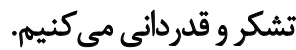

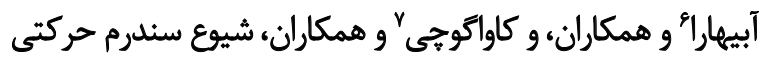

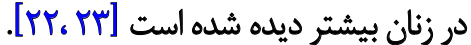

در اين مطالعه مشخص شد، افراد با سابقه مثبت درد مزمن (4) بردئ

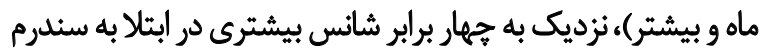

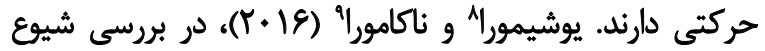

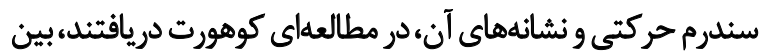

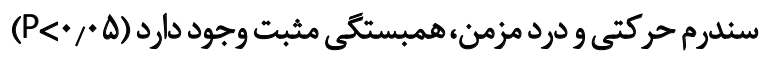

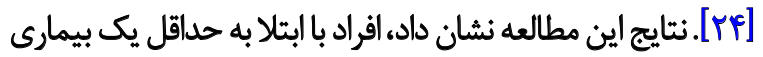

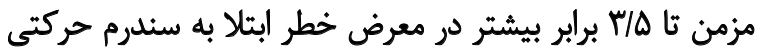

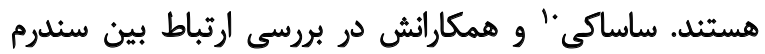

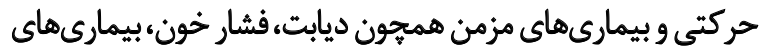

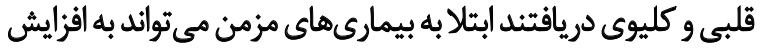

احتمال ابتلا به سندرم حركتى منجر شود [Yهافئل

همجنين در مطالعهاى باعنوان لابررسى اختلالات كارديومتابوليكي

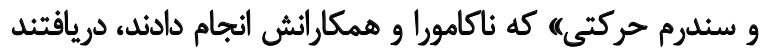

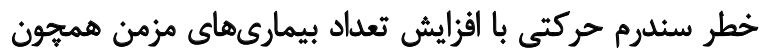

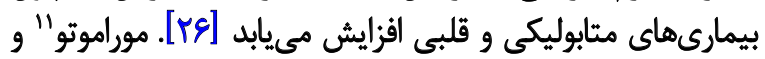

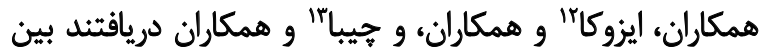

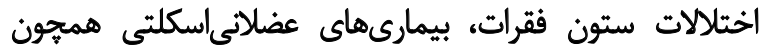

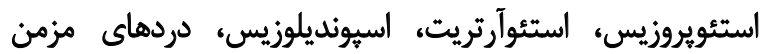

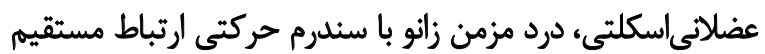

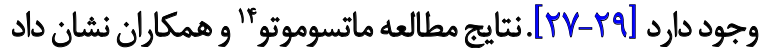

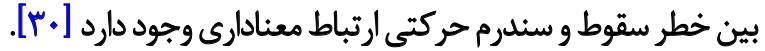

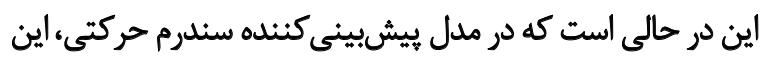

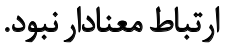

\section{نتيجه كيرى نهمايبى}

با توجه به شيوع زياد سندرم حركتى در جمعيت سالمند و

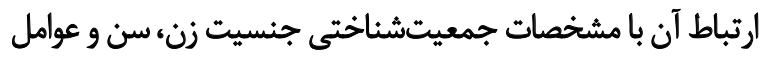

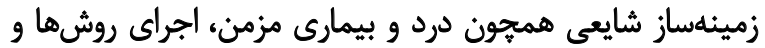

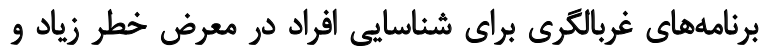

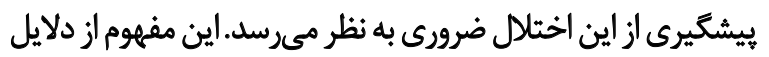

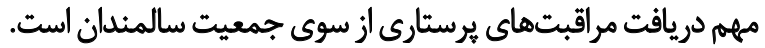
از طرفى با وجود تبعات زياد سندرم حركتى در دوره سالمندى، به به ساني

\section{Ebihara}

7. Kawaguchi

8. Youshimura

9. Nakamura

10. Sasaki

11. Muramoto

12. lizuka

13. Chiba

14. Matsumoto 


\section{References}

[1] Asgarifar G. [Disease of aging (Persian)]. Tehran: University of social welfare and rahabilitation science; 2016.

[2] Mirzaei M, GHahfarookhi M. [The demography of elderly people in Iran based on the counts from 1956-2006 (Persian)]. Iranian Journal of Ageing. 2008; 2(5):326-31.

[3] Haghshenas N. Sociological aspects and population aging (Persian)]. Journal of Sociological Studies. 2012; 1(2):133-47.

[4] Mousavi A, VaezmousavI M, Zehtab-Najafi A. [Effects of Yoga techniques on reducing mental symptoms of immobility syndrome in elderly women. Journal of Rehabilitation Medicine (Persian)]. 2014; 2(4):37-45.

[5] Akai M, Doi T, Seichi A, Okuma Y, Ogata T, Iwaya T. Locomotive syndrome: Operational definition based on a questionnaire, and exercise interventions on mobility dysfunction in elderly people. Clinical Reviews in Bone and Mineral Metabolism. 2016; 14(2):119-31. [DOI:10.1007/s12018-016-9210-8] [PMID] [PMCID]

[6] Nakamura K, Ogata T. Locomotiv syndrome: Definition and management. Clinical Reviews in Bone and Mineral Metabolism. 2016; 14(2):56-67. [DOI:10.1007/s12018-016-9208-2] [PMID] [PMCID]

[7] Nakamura K. The concept and treatment of locomotive syndrome: its acceptance and spread in Japan. Journal of Orthopaedic Science. 2011; 16(5):489-91. [DOI:10.1007/s00776-011-0108-5] [PMID] [PMCID]

[8] Klussmann A, Gebhardt H, Nübling M, Liebers F, Quirós Perea E, Cordier W. Individual and occupational risk factors for knee osteoarthritis: results of a case-control study in Germany. Arthirirts Research and Therapy. 2010; 12(3):R88. [DOI:10.1186/ar3015].

[9] Tavares DRB, Santos FC. Locomotive syndrome in the elderly: translation, cultural adaptation, and Brazilian validation of the tool 25-Question Geriatric Locomotive Function Scale. Revista Brasileira de Reumatologia. 2017; 57(1):56-63. [DOI: 10.1016/j.rbr].

[10] Makino T, Kaito T, Yonenobu K. Spinal disorders as a cause of locomotive syndrome: The influence on functional mobility and activities of daily living. Clinical Reviews in Bone and Mineral Metabolism. 2016; 14(2):105-15. [DOI:10.1007/s12018-016-9213-5]

[11] Hashizume H, Noriko, Yoshimura, Nagata K, Miyazaki N, Ishimoto Y, et al. Development and evaluation of a video exercise program for locomotive syndrome in the elderly. Modern Rheumatology Journal. 2014; 24(2):250-7. [DOI:10.3109/14397595.2013. 854063] [PMID]

[12] Ishijima M, Kaneko H, Hada S, Kinoshita M, Sadatsuki R, Liu $\mathrm{L}$, et al. Osteoarthritis as a cause of locomotive syndrome: Its influence on functional mobility and activities of daily living. Clinical Reviews in Bone and Mineral Metabolism. 2016; 14(2):77-104. [DOI:10.1007/s12018-016-9212-6]

[13] Antonopoulou MD, Alegakis AK, Hadjipavlou AG, Lionis $\mathrm{CD}$. Studying the association between musculoskeletal disorders, quality of life and mental health. A primary care pilot study in rural Crete, Greece. BMC Musculoskelet Disord. 2009; 10(1):143. [DOI:10.1186/1471-2474-10-143] [PMID] [PMCID]

[14] Ikemoto T, Arai YC. Locomotive syndrome: Clinical perspectives. Clinical Interventions in Aging. 2018; 13:819-27. [DOI:10.2147/CIA.S148683] [PMID] [PMCID]
[15] Seichi A, Hoshino Y, Doi T, Akai M, Tobimatsu Y, Iwaya T. Development of a screening tool for risk of locomotive syndrome in the elderly: The 25-question Geriatric Locomotive Function Scale. Journal of Orthopaedic Science. 2012; 17(2):163-72. [DOI:10.1007/ s00776-011-0193-5] [PMID]

[16] Akahane M, Maeyashiki A, Tanaka Y, Imamura T. The impact of musculoskeletal diseases on the presence of locomotive syndrome. Modern Rheumatology. 2018; 29(1):151-6. [DOI: 10.1080/ 14397595.2018.1452173].

[17] Hirano K, Imagama S, Hasegawa Y, Ito Z, Muramoto A, Ishiguro N. The influence of locomotive syndrome on health-related quality of life in a community-living population. Modern Rheumatology. 2013; 23(5):939-44. [DOI:10.3109/s10165-012-0770-2] [PMID]

[18] Hoshino Y, Seichi A. Locomotive syndrome and frailty. Locomotive Syndrome in the super-aged society. Clinical calcium. 2012; 22(4):21-6. [PMID]

[19] Sadeghi mahalli N, Hosseini M, Rahgozar M, NouroziTabrizi K. [Evaluation of cultural adaptation, validity and reliability of the questionnaire of geriatric locomotive function scale-25 questions (Persian)]. Pharmacophore. 2017; 8(6s):1-5.

[20] Nakamura K. A "super-aged" society and the "locomotive syndrome". Journal of Orthopedic Science. 2008; 13(1):1-2. [DOI:10.1007/s00776-007-1202-6] [PMID] [PMCID]

[21] Kimura A, Seichi A, Konno S, Yabuki S, Hayashi K. Prevalence of locomotive syndrome in Japan: A nationwide, cross sectional Internet survey. Journal of Orthopaedic Science. 2014; 19(5):792-7. [DOI:10.1007/s00776-014-0606-3] [PMID]

[22] Ebihara C, Arai T, Fujita H, Katou g, Sinooka S, Morita Y, et al. The relationship between locomotive syndrome and quality of life of community-dwelling elderly people. Rigakuryoho Kagak. 2013; 28(5):569-72. [DOI:10.1589/rika.28.569]

[23] Kawaguchi H. Locomotive syndrome. Japanese Journal of Geriatrics. 2014; 51(2):123-5. [DOI:10.3143/geriatrics.51.123] [PMID]

[24] Yoshimura N, Nakamura K. Epidemiology of locomotive organ disorders and symptoms: An estimation using the population-based cohorts in Japan. Clinical Reviews in Bone and Mineral Metabolism. 2016; 14(2):68-73. [DOI:10.1007/s12018-016-9211-7] [PMID] [PMCID]

[25] Sasaki E, Ishibashi Y, Tsuda E, Ono A, Inoue YY, Takahashi I, et al. Evaluation of locomotive disability using loco-check: Acrosssectional study in the Japanese general population. Journal of Orthopaedic Science. 2012; 18(1):121-9. [DOI:10.1007/s00776-0120329-2] [PMID]

[26] Nakamura M, Kobashi Y, Hashizume H, Oka H, Kono R, Nomura S, et al. Locomotive syndrome is associated with body composition and cardiometabolic disorders in elderly Japanese women. BioMed Central. 2016; 27; 16:166. [DOI: 10.1186/s12877016-0339-6].

[27] Muramoto A, Imagama S, Ito Z, Ishiguro N, Hasegawa Y. Spinal sagittal balance substantially influences locomotive syndrome and physical performance in community-living middle-aged and elderly women. Journal of Orthopaedic Science. 2016; 21(2):126221. [DOI:10.1016/j.jos.2015.12.016] [PMID]

[28] Chiba D, Tsuda E, Wada K, Kumagai G, Sasaki E, Nawata A. Lumbar spondylosis, lumbar spinal stenosis, knee pain, back muscle strength are associated with the locomotive syndrome: 
Rural population study in Japan. Journal of Orthopaedic Science. 2016; 21(3):366-72. [DOI:10.1016/j.jos.2016.02.006] [PMID]

[29] lizuka Y, lizuka H, Mieda T, Tajika T, Yamamoto A, Takagishi K. Population-based study of the association of osteoporosis and chronic musculoskeletal pain and locomotive syndrome: The Katashina study. Journal of Orthopaedic Science. 2015; 20(6):10859. [DOI:10.1007/s00776-015-0774-9] [PMID]

[30] Matsumoto H, Hagino H, Wada T, Kobayashi E. Locomotive syndrome presents a risk for falls and fractures in the elderly Japanese population. Osteoporosis and Sarcopenia. 2016; 2(3):156-63. [DOI:10.1016/j.afos.2016.06.001] 